\title{
Inheritance and Linkage of Thirteen Polymorphic Isozyme Loci in Diploid Blueberry
}

\author{
M.I. van Heemstra, L.P. Bruederle', and N. Vorsa ${ }^{2}$ \\ Blueberry and Cranberry Research Center, New Jersey Agricultural Experiment Station, Rutgers \\ University, Chatsworth, NJ 08019 \\ Additional index words. Vaccinium, allozymes, segregation
}

\begin{abstract}
Diploid populations and progenies of controlled crosses of blueberry, Vaccinium section Cyanococcus, were analyzed by starch gel electrophoresis for nine enzyme systems, aconitase (ACO), aldolase (ALD), alcohol dehydrogenase (ADH), glyceraldehyde-3 -phosphate dehydrogenase (G-3 -PDH), isocitrate dehydrogenase (IDH), leucine aminopeptidase (LAP), malate dehydrogenase (MDH), 6-phosphogluconate dehydrogenase (6-PGD), and phosphoglucose isomerase (PGI). Allozyme variants indicated the existence of three alleles at Ace-1, Ald, G-3-pdh-2, and Mdh-2; four alleles at Mdh-3 and 6-Pgd-2; five alleles at Aco-2, Idh, Lap-1, and 6-Pgd-1; six alleles at Adh-2 and Pgm-2; and nine alleles at Pgi-2. In addition, a null allele was found at Lap-1. In the majority of progenies, the inheritance patterns for each of these loci were consistent with mendelian laws for single gene control. Forty-seven pairs of loci were tested for independent assortment revealing two linked pairs, Pgi-2/Lap-1 and Pgm-2/6-Pgd-2, which appeared to represent two independent linkage groups.
\end{abstract}

An extensive survey and discussion of Vaccinium section Cyanococcus A. Gray in North America by starch gel electrophoresis has revealed high levels of variability (Bruederle and Versa, 1989; Bruederle et al., 1991); 90\% of the allozyme loci examined were polymorphic within the section. Inheritance studies, however, have been reported for only a few loci; Versa et al. (1988) presented evidence for mendelian inheritance at four loci among the enzyme systems malate dehydrogenase (EC 1.1.1.37; $\mathrm{MDH}$ ) phosphoglucose isomerase (EC 5.3.1.9; PGI) 6-phosphogluconate dehydrogenase (EC 1.1.1.44; 6-PGD), and isocitrate dehydrogenase (EC 1.1.1.42; IDH); Krebs and Hancock (1989) reported tetrasomic inheritance at four loci among the enzyme systems MDH, PGI, 6-PGD, and aspartate aminotransferase (EC 2.6.1.1). Evidence for the genetic basis of more isozyme phenotypes and knowledge of the linkage relationships among isozyme loci would be useful for future genetic and breeding behavior studies and provide information on linkage conservation. The Vaccinium section Cyanococcus karyotype consists of 12 metacentric or submetacentric chromosomes (Hall and Galletta, 1971) that will potentially result in the development of maps for 12 linkage units.

The purpose of this paper is to report: i) inheritance patterns at the diploid level for nine of the isozyme loci governing the enzyme systems aconitase (EC 4.2.1.3; ACO), alcohol dehydrogenase (EC 1.1.1.1; ADH), aldolase (EC 4.1.2.13; ALD), glyceraldehyde-3 -phosphate dehydrogenase (EC 1.2.1.9; G-3$\mathrm{PDH}$ ), leucine aminopeptidase (EC 3.4.11.1; LAP) MDH, 6PGD, and phosphoglucomutase (EC 2.7.5.1; PGM); (ii) linkage relationships among 13 of the loci governing these enzyme systems as well as IDH and PGI; and (iii) allozyme variants observed at these loci in $V$. boreale Hall and Aalders, $V$. myrtilloides Mich., V. corymbosum L., V. pallidum Ait., V. tenellum Ait., and $V$. darrowi Camp. in order to update locus and allelic nomenclature in Vaccinium section Cyanococcus.

\footnotetext{
Received for publication 19 Jan. 1990. This research was supported by State funds, New Jersey Agricultural Experiment Station, Publication no. D-1216314-89, the U.S. Hatch Act, and USDA-ARS Cooperative Agreement no. 583615-7-007. The cost of publishing this paper was defrayed in part by the payment of page charges. Under postal regulations, this paper therefore must be hereby marked advertisement solely to indicate this fact.

'Biology Dept., The Univ. of Michigan-Flint, Flint, MI 48502-2186.

${ }^{2}$ Author to whom correspondence should be addressed.
}

\begin{abstract}
Materials and Methods
Plant material. Thirty diploid blueberry populations collected as cuttings throughout eastern North America from Nova Scotia to central Florida were surveyed for allelic variants. The populations ranged from 25 to 55 individuals and included the six diploid species of Vaccinium section Cyanococcus (sensu Vander Kloet 1988): V. boreale, V myrtilloides, V. corymbosum, $V$. pallidum, $V$. tenellum, and $V$. darrowi.
\end{abstract}

For inheritance studies, crosses were made using the diploid plants described in Versa et al. (1988). The plants labeled "dar-" and "en-" belong to the $V$. darrowi and $V$. elliottii (sensu Chap.) populations described in that study. US 392,84216, and 842-29 are progeny of Fla4b (a selected wild clone from Ocala National Forest, Fla.) crossed with an unknown plant. Flowers were emasculated before pollination.

Electrophoresis. Vegetative buds were ground in a buffer consisting of $0.1 \mathrm{M}$ sodium phosphate containing $10 \% \mathrm{w} / \mathrm{V}$ polyvinylpyrrolidone (PVP-40) and 1\% 2-mercaptoethanol at pH 7.5; extracts were absorbed onto Whatman no. 17 chromatography paper wicks $(2 \times 10 \mathrm{~mm})$. About one vegetative bud per drop of buffer and per wick was used. Wicks were then stored in microcentrifuge tubes at $-72 \mathrm{C}$ until electrophoresis. Wicks were loaded onto $12 \%$ starch gels and electrophoresis was performed in a refrigerator maintained at $2 \mathrm{C}$.

The gel and electrode buffer systems used were the Soltis et al. (1983) system 7 for LAP-1, ADH-2, and PGI-2 at $250 \mathrm{~V}$ (constant); system 8 for ACO-1, ACO-2, ADH-2, ALD, LAP1 , and PGI-2 at $250 \mathrm{~V}$ (constant); system 1 modified to pi-1 7.5 for ALD, G-3-PDH-2, IDH, 6-PGD-2, and PGM-2 at $100 \mathrm{~V}$ (constant); and system 11 modified to $\mathrm{pH} 6.5$ for ACO-1, ACO2, G-3-PDH-2, MDH-2, MDH-3, 6-PGD-1, and PGM-2 at 55 $\mathrm{V}$ (constant). The pH 6.1 morpholine citrate gel and electrode buffer system of Clayton and Tretiak (1972) was used for IDH, MDH-2, MDH-3, 6-PGD-1, and 6-PGD-2 at $120 \mathrm{~V}$ (constant). Whereas population genetic data were collected employing all of the aforementioned systems, progenies of controlled crosses were run on two or three of the systems depending on which loci were expected to segregate.

Gel slices $1 \mathrm{~mm}$ thick were stained for the appropriate enzymes. G-3-PDH was stained according to the following procedure: $500 \mathrm{mg}$ fructose 1,6-diphosphate trisodium salt was incubated with 30 units of aldolase in $20 \mathrm{ml}$ of distilled water at $37 \mathrm{C}$ for 30 rein, after which $50 \mathrm{ml}$ distilled water, $2 \mathrm{ml} 1.0$ 
M arsenic acid, $40 \mathrm{mg}$ NADP, $20 \mathrm{mg}$ 3-(4,5 -Dimethylthiazol2-yl)-2,5-diphenyltetrazolium bromide (MTT), and $5 \mathrm{mg}$ of phenazine methosulfate were added. The other enzymes were stained according to Soltis et al. (1983) with the following modifications: For ACO, the cis-aconitic acid was increased to 300 $\mathrm{mg}$ and the isocitrate dehydrogenase to 60 units. For LAP, the gel slice was first incubated with the substrate for $30 \mathrm{~min}$ and rinsed off before addition of fast black $\mathrm{K}$ salt dissolved in 1.0 M phosphate buffer at $\mathrm{pH}$ 6.0.

The migration distance of isozyme bands was measured and their mobility calculated in relation to the buffer front. The mobilitie; presented in Fig. 1 were measured on: system 7 (ADH2, LAP-1, PGI-1, and PGI-2); system 1 at $\mathrm{pH} 7.5$ (IDH and ALD); system 11 at $\mathrm{pH} 6.5$ (ACO-1, ACO-2, PGM-1, PGM2, G-3-PDH-1, and G-3-PDH-2); and on the morpholine citrate system (MDH-2, MDH-3, 6-PGD-1, and 6-PGD-2). The loci for a given enzyme were assigned sequential numbers starting with 1 for the most anodal locus; the allozymes found were assigned sequential letters starting with a for the most anodal. Any null alleles found were referred to as $n$.

Data analysis. $G$ statistics for goodness-of-fit to expected ratios were calculated for single locus inheritance analyses (Sokal and Rohlf, 1981). Pooled and heterogeneity G statistics were calculated for progeny segregating for identical alleles. The correction for continuity for cases in which there was only one degree of freedom was used only for progeny in which a difference in alleles precluded pooling with other progeny.

Contingency table $\mathrm{G}$ statistics were calculated to test for independent assortment in progeny jointly segregating for two loci. Recombination values for cases of significant deviation from independent assortment were obtained from the LINKAGE-1 computer program of Suiter et al. (1983). Allozyme segregations were not included in the analysis if any of their expected frequency classes were below five. Linkage analyses in which the segregation ratio of one out of the two loci differed significantly from the expected mendelian ratio were accepted.

\section{Results}

The allozyme variants reported here are discussed with respect to their migration distances, quaternary structure, and inheritance only. A discussion of the distribution of allozyme polymorphism among the species was not considered relevant to this paper and are discussed elsewhere (Bruederle and Versa, 1990; Bruederle et al., 1991).

Malate dehydrogenase. In addition to the two previously reported MDH loci (Versa et al., 1988), two weakly staining zones, one anodal and one cathodal to the former, were detected as a result of generally improved isozyme resolution. Assuming these are additional MDH loci, the former MDH-1 and MDH2 would then become MDH-2 and MDH-3 and will subsequently be referred to as such. MDH-1 was monomorphic, consisting of one band at the same migration distance in all individuals analyzed. MDH-4 was too faint to be scored. One additional allele was found at $M d h-3$ at a migration distance between the $a$ and $b$ alleles reported in a former study (Versa et al., 1988) in which mendelian inheritance for this locus was reported.

Except for a few individuals that are discussed below, banding patterns at $M d h-2$ were either single-banded or three-banded, the center band being darker and about equidistant from the peripheral bands in accordance with a dimeric structure of the enzyme. Banding positions were consistent with the existence of three alleles at this locus (Fig. 1). Progenies segregating for Mdh-2 supported mendelian inheritance (Table 1).

In certain individuals, there also were extra bands observed in proximity to the $b$ allele of $M d h-2$, the genetic basis of which cannot be determined conclusively with the present data. One pattern consisted of the $b$ allele with a weaker cathodal band; another pattern consisted of the $b$ allele with a weaker anodal band (Fig. 2). These patterns were reproducible and appeared to segregate in a mendelian fashion. A cross between two individuals homozygous for the $b$ allele but with one having the additional cathodal band gave a progeny segregation consistent with a 1:1 ratio for the parental patterns. A cross between two individuals with the $M d h-2 b$ allele, one parent possessing the additional anodal band and

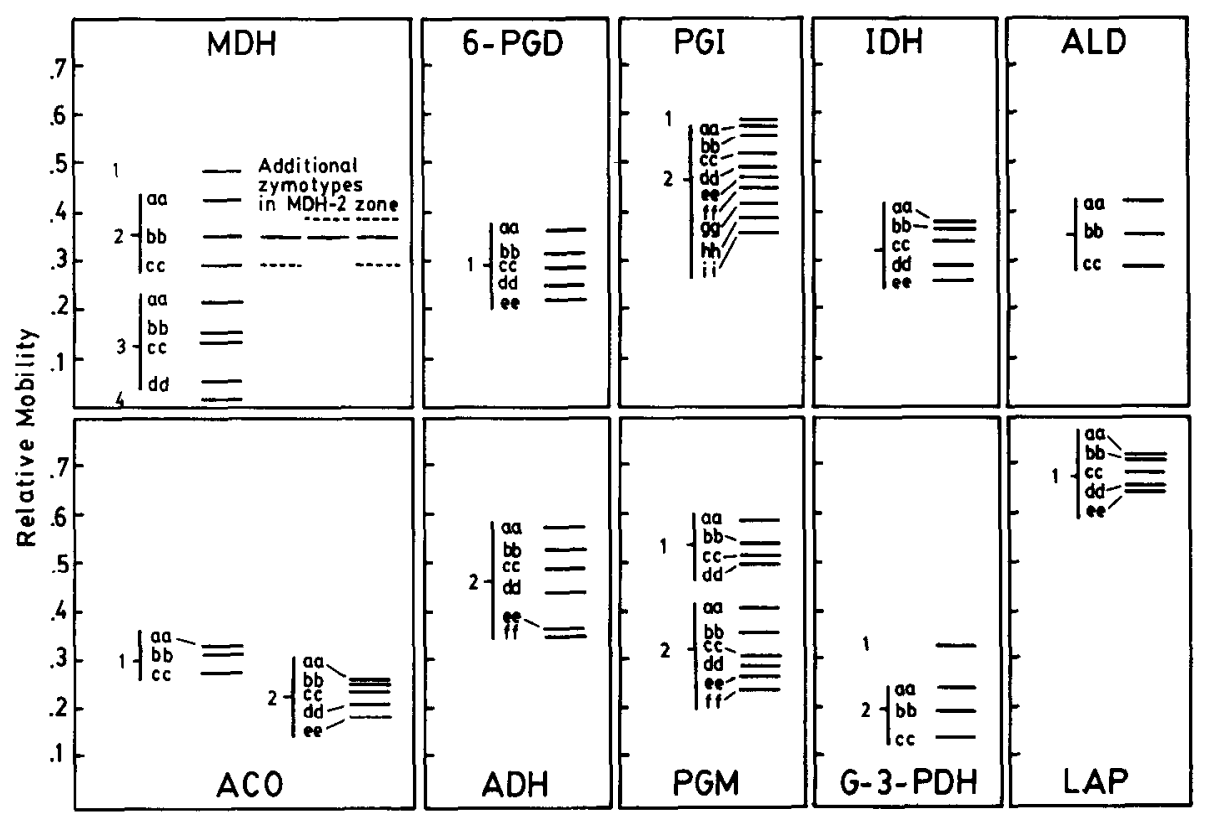

Fig. 1. Electrophoretic mobility of Vaccinium section Cyanococcus isozymes (solid lines). Numbers indicate loci. Letters indicate alleles. Dotted lines indicate bands with a weaker staining intensity relative to the solid bands. 
Table 1. Single-locus goodness-of-fit tests (G statistics) at nine polymorphic isozyme loci in Vaccinium section Cyanococcus.

\begin{tabular}{|c|c|c|c|c|c|c|}
\hline Locus & Cross $^{z}$ & Parental genotypes & Progeny genotypes & Expected ratio & df & $\mathrm{G}$ \\
\hline$A c O-1$ & $\begin{array}{l}2 \\
7\end{array}$ & $\begin{array}{l}a b \times b b \\
b b \times a b\end{array}$ & $\begin{array}{l}15 a b: 19 b b \\
22 a b: 17 b b\end{array}$ & $\begin{array}{c}1: 1 \\
1: 1 \\
G \text { pooled } \\
G \text { heterogeneity }\end{array}$ & $\begin{array}{l}1 \\
1 \\
1 \\
1\end{array}$ & $\begin{array}{l}0.47 \\
0.64 \\
0.01 \\
1.10\end{array}$ \\
\hline Aco-2 & $\begin{array}{l}2 \\
6 \\
7\end{array}$ & $\begin{array}{l}a b \times a a \\
a b \times a b \\
b b \times a b\end{array}$ & $\begin{array}{c}41 a a: 23 a b \\
10 a a: 24 a b: 14 b b \\
30 a b: 27 b b\end{array}$ & $\begin{array}{l}1: 1 \\
1: 2: 1 \\
1: 1\end{array}$ & $\begin{array}{l}1 \\
2 \\
1\end{array}$ & $\begin{array}{l}4.57^{*} \\
0.67 \\
0.16\end{array}$ \\
\hline Adh-2 & $\begin{array}{r}1 \\
2 \\
7 \\
14\end{array}$ & $\begin{array}{l}b b \times b d \\
b d \times b b \\
b b \times b d \\
b b \times b d\end{array}$ & $\begin{array}{l}21 b b: 26 b d \\
34 b b: 34 b d \\
38 b b: 40 b d \\
17 d d: 17 b d \\
39 b d: 23 d d .\end{array}$ & $\begin{array}{c}1: 1 \\
1: 1 \\
1: 1 \\
1: 1 \\
\mathrm{G} \text { pooled } \\
\mathrm{G} \text { heterogeneity } \\
1: 1\end{array}$ & $\begin{array}{l}1 \\
1 \\
1 \\
1 \\
1 \\
3 \\
1\end{array}$ & $\begin{array}{l}0.53 \\
0.00 \\
0.05 \\
0.00 \\
0.21 \\
0.37 \\
3.66\end{array}$ \\
\hline Ald & $\begin{array}{r}1 \\
2 \\
12 \\
14\end{array}$ & $\begin{array}{l}b c \times b b \\
b b \times b c \\
b b \times b c \\
b c \times b b \\
b c \times b c\end{array}$ & $\begin{array}{c}40 b b: 52 b c \\
39 b b: 55 b c \\
48 b b: 44 b c \\
24 b b: 29 b c \\
29 b b: 47 b c: 23 c c\end{array}$ & $\begin{array}{c}1: 1 \\
1: 1 \\
1: 1 \\
1: 1 \\
\text { G pooled } \\
\text { G heterogeneity } \\
1: 2: 1\end{array}$ & $\begin{array}{l}1 \\
1 \\
1 \\
1 \\
1 \\
3 \\
2\end{array}$ & $\begin{array}{l}1.57 \\
2.74 \\
0.17 \\
0.47 \\
2.54 \\
2.41 \\
0.95\end{array}$ \\
\hline$G 3 p d h-2$ & $\begin{array}{l}1 \\
2 \\
5\end{array}$ & $\begin{array}{l}b b \times b c \\
b c \times b b \\
b b \times b c \\
a b \times a b\end{array}$ & $\begin{array}{c}34 b b: 47 b c \\
57 b b: 41 b c \\
45 b b: 51 b c \\
21 a a: 44 a b: 28 b b\end{array}$ & $\begin{array}{c}1: 1 \\
1: 1 \\
1: 1 \\
\text { G pooled } \\
\text { G heterogeneity } \\
1: 2: 1\end{array}$ & $\begin{array}{l}1 \\
1 \\
1 \\
1 \\
2 \\
2\end{array}$ & $\begin{array}{l}2.09 \\
2.62 \\
0.37 \\
0.03 \\
5.06 \\
1.27\end{array}$ \\
\hline Lap-1. & $\begin{array}{r}6 \\
10 \\
11 \\
12 \\
14\end{array}$ & $\begin{array}{l}a c \times b n \\
b d \times d d \\
a c \times b b \\
a c \times c c \\
a b \times c n\end{array}$ & $\begin{array}{c}39 a b: 37 b c: 27 a n: 17 c n \\
61 b d: 74 d d \\
34 a b: 34 b c \\
28 a c: 24 c c \\
9 a n: 15 a c: 14 a n: 14 b n\end{array}$ & $\begin{array}{c}1: 1: 1: 1 \\
1: 1 \\
1: 1 \\
1: 1 \\
1: 1: 1: 1\end{array}$ & $\begin{array}{l}3 \\
1 \\
1 \\
1 \\
3\end{array}$ & $\begin{array}{c}10.98^{*} \\
1.07 \\
0.00 \\
0.17 \\
1.82\end{array}$ \\
\hline$M d h-2$ & $\begin{array}{l}12 \\
13 \\
14\end{array}$ & $\begin{array}{l}b b \times b c \\
b b \times b c \\
b b \times b c\end{array}$ & $\begin{array}{l}36 b b: 33 b c \\
41 b b: 59 b c \\
31 b b: 21 b c\end{array}$ & $\begin{array}{c}1: 1 \\
1: 1 \\
1: 1 \\
\text { G pooled } \\
\text { G heterogeneity }\end{array}$ & $\begin{array}{l}1 \\
1 \\
1 \\
1 \\
2\end{array}$ & $\begin{array}{l}0.13 \\
3.26 \\
1.93 \\
0.11 \\
5.21\end{array}$ \\
\hline $6-P g d-1$ & $\begin{array}{l}3 \\
4 \\
9\end{array}$ & $\begin{array}{l}a b \times a b \\
a b \times a b \\
a b \times a b\end{array}$ & $\begin{array}{c}9 a a: 19 a b: 9 b b \\
24 a a: 32 a b: 14 b b \\
30 a a: 41 a b: 13 b b\end{array}$ & $\begin{array}{c}1: 2: 1 \\
1: 2: 1 \\
1: 2: 1 \\
\text { G pooled } \\
\text { G heterogeneity } \\
1: 1 \\
1: 1 \\
\text { G pooled } \\
G \text { heterogeneity } \\
1: 1: 1: 1\end{array}$ & $\begin{array}{l}2 \\
2 \\
2 \\
2 \\
4 \\
1 \\
1 \\
1 \\
1 \\
3\end{array}$ & $\begin{array}{l}0.03 \\
3.18 \\
6.95^{*} \\
7.71^{*} \\
2.45 \\
0.17 \\
0.05 \\
0.02 \\
0.20 \\
2.36\end{array}$ \\
\hline Pgm-2 & $\begin{array}{l}1 \\
2 \\
3 \\
4\end{array}$ & $\begin{array}{l}a b \times b b \\
b b \times a b \\
a b \times b b \\
a b \times b b\end{array}$ & $\begin{array}{l}38 a b: 55 b b \\
45 a b: 54 b b \\
24 a b: 14 b b \\
48 a b: 43 b b\end{array}$ & $\begin{array}{c}1: 1 \\
1: 1 \\
1: 1 \\
1: 1 \\
\text { G pooled } \\
\text { G heterogeneity } \\
1: 2: 1 \\
1: 2: 1 \\
\text { G pooled } \\
\text { G heterogeneity } \\
1: 1\end{array}$ & $\begin{array}{l}1 \\
1 \\
1 \\
1 \\
1 \\
3 \\
2 \\
2 \\
2 \\
2 \\
1\end{array}$ & $\begin{array}{l}3.12 \\
0.82 \\
2.66 \\
0.27 \\
0.38 \\
6.50 \\
4.68 \\
5.75 \\
6.60^{*} \\
3.82 \\
0.58\end{array}$ \\
\hline
\end{tabular}

${ }^{2}$ Crosses are: dar20 $\times$ dar18 (1); dar18 $\times$ dar20 (2); 842-16 $\times$ Ten (3); 842-29 $\times$ Ten (4); dar20 $\times$ dar22 (5); ellFL6 $\times$ dar16 (6); dar10 $\times$ dar18 (7); ellFL19 $\times$ W85-6(8); US392 $\times$ US136 (9); dar14 $\times$ ellNC19 (10); ellFL6 $\times$ dar10 (11); ellFL6 $\times$ dar12 (12); ellFL19 $\times$ dar4 (13); dar5 $\times$ dar6 (14).

*Significant at $P=0.05$. 


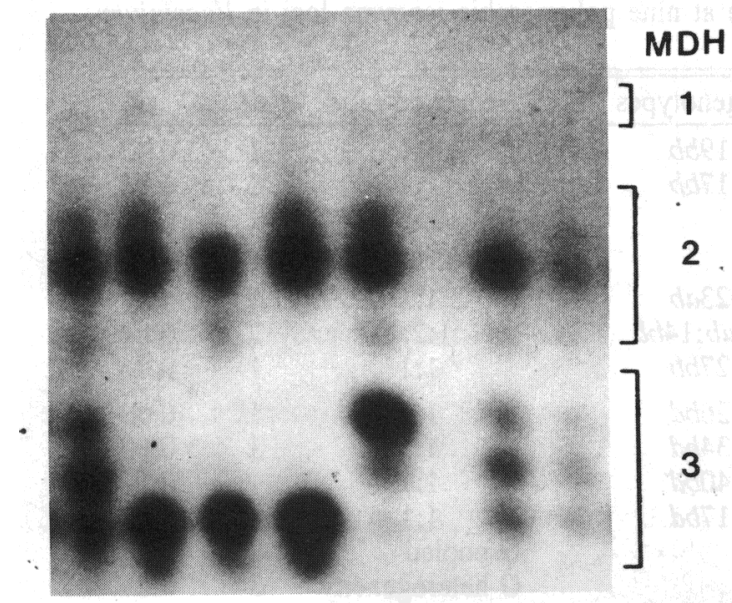

Fig.2. MDH zymograms showing additional variation found in proximity of MDH-2. From left to right the first three genotypes are interpreted to be: (1) Mdr-2bb with both extra anodal and cathodal bands, Mdh-3ac; (2) Mdh-2bb with extra anodal band, Mdh-3cc; (3) $M d h-2 b b$ with extra cathodal band, $M d h-3 c c$.

the other the additional cathodal band, segregated in a 1:1:1:1 fashion for four patterns: the $M d h-2 b b$ allozyme alone, the $M d h-2 b b$ allozyme with both extra anodal and cathodal bands, and the two parental patterns. The $M d h-2 b b$ band always stained with the same intensity in the parents and in the progeny whether or not the extra anodal and/or cathodal bands were present. A cross between a plant exhibiting only the $M d h-2 b b$ band and a plant with a three-banded $M d h-2 b c$ pattern gave progeny segregating in a 1:1 ratio for the three-banded $M d h$ $2 b c$ pattern and the $M d h-2 b b$ band with the additional cathodal band. A cross between a plant possessing the $M d h-2 b b$ band with the extra cathodal band and a plant with the threebanded pattern $M d h-2 b c$ resulted in three types of progeny: 14 exhibited only the $M d h-2 b b$ band, 18 had the $M d h-2 b c$ bands with the additional cathodal band, and 21 had the threebanded $M d h-2 b c$ pattern. One hypothesis that is consistent with the data would be the existence of another MDH locus that produced a monomeric enzyme with three alleles comigrating with $M d h-2$. The $b b$ allozyme of this hypothesized locus would co-migrate with the $M d h-2 b b$ band, the $a a$ allozyme would be the extra anodal band, and the $c c$ allozyme would be the extra cathodal band that would co-migrate with $M d h-2 c c$. Although MDH has usually been found to be a dimer, two-banded MDH phenotypes have been reported in organelles of other plants (Longo and Scandalios, 1969; Sternberg and Ting, 1979). The subcellular localization of the various blueberry MDH loci has not been done and may help to explain these observations.

6-phosphogluconate dehydrogenase. Progeny segregations for 6-Pgd-1 gave either one-banded or three-banded patterns consistent with a dimeric structure for the enzyme. Banding positions suggested the existence of five alleles at 6-Pgd-1 (Fig. 1). Segregation ratios were in accordance with mendelian inheritance (Table 1) except for cross 9. Mendelian inheritance for 6$P g d-2$ has already been shown (Versa et al., 1988); no new alleles were found at this locus.

Phosphoglucose isomerase. Mendelian inheritance has been reported for $P g i-2$ (Versa et al., 1988). In that study, one pattern obtained for Pgi-2 contained only two discernible bands, the third band having been hypothesized to co-migrate with PGI-1.
This hypothesis has now been confirmed; with electrophoresis

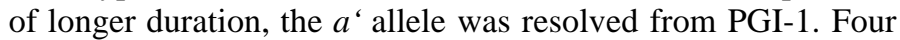
new alleles were identified, thus converting the former Pgi-2 $a^{\prime}, a, b, c$, and $d$ alleles to Pgi-2 $a, b, d, e$, and $\mathrm{g}$ alleles.

Isocitrate dehydrogenase. Mendelian inheritance for Idh has already been published (Versa et al., 1988). Two additional ldh alleles were found, one with a migration distance between the former $a$ and $b$ alleles and the other migrating between the former $b$ and $\mathrm{c}$ alleles. Thus, the former $I d h-b$ and $\mathrm{c}$ alleles become $l d h-c$ and e alleles, respectively.

Aldolase. Staining for ALD showed one zone of activity with either one-banded or five-banded electrophoretic patterns. This result is consistent with a tetrameric structure for this enzyme in blueberry. Banding positions indicated the existence of three alleles (Fig. 1). Segregation ratios were in accordance with expected ratios (Table 1) for single locus control.

Aconitase. Two zones of staining for aconitase could be distinguished; banding patterns for each zone consisted of either one or two bands. This observation is consistent with a monomeric structure for both Aco-1 and Aco-2 (Fig. 1). ACO has been found to be a monomer in other plant species (Griffin and Palmer, 1987; Hyun et al., 1987). Banding positions in blueberry indicated the existence of three alleles at Ace-1 and five at Aco-2. Staining for ACO in the progeny analyses was faint, making it more difficult to read than the other enzymes. Therefore, progeny numbers for segregation and linkage analyses were generally reduced compared to other enzymes. However, the available data support mendelian inheritance (Table 1).

Alcohol dehydrogenase. ADH exhibited two zones of activity, the first of which was very weak. The second zone, although sometimes weak in leaf tissue, was storable; banding patterns consisted of either one or three bands in agreement with a dimeric structure for Adh-2. $\mathrm{ADH}$ has been found to be a dimer in other plant species (Suiter, 1988; Weeden and Marx, 1987). Six alleles were found at Adh-2 in blueberry (Fig. 1). Segregation ratios were in accordance with mendelian inheritance (Table 1).

Phosphoglucomutase. In general, PGM showed two zones of activity, the zymotypes for each zone being either one-banded or two-banded; this pattern is consistent with a monomeric structure for Pgm-1 and Pgm-2. PGM has been found to be a monomer in many plant species (Arus et al., 1982, Hyun et al., 1987; Manes and Fairbrothers, 1987; Suiter, 1988). Pgm-1 will not be discussed here as we are not confident in the scoring of the progeny data 'for this locus. Six alleles were found at Pgm-2 (Fig. 1). Progenies with expected segregation ratios of $1: 1$ for Pgm-2 did not deviate significantly from the expected ratio but progenies with expected segregation ratios of 1:2:1 did (Table 1 ).

Glyceraldehyde-6-phosphate dehydrogenase. Two zones of activity for G-3-PDH could be distinguished. G-3-pdh-1 was monomorphic and consisted of one band in all species and progenies analyzed. G-3-pdh-2 was polymorphic, zymotypes being either single-banded or expressed as a diffuse zone of staining extending between the migration distances of two of the single bands. The diffuse zone was interpreted to represent the closely migrating bands of heterozygotes for a multimeric enzyme, and the migration distance of the zymotypes found was consistent with the existence of three alleles (Fig. 1). Following this interpretation, segregation ratios calculated for progeny were in accordance with mendelian inheritance (Table 1).

Leucine aminopeptidase. Two zones of activity were observed, the second zone being too faint to be scored. Zymotypes found for Lap-1 were either one-banded or two-banded, consis- 
Table 2. Number (and size) of progenies analyzed by G statistics contingency tests for independent assortment of jointly segregating isozyme loci (above the diagonal) and number of progenies deviating from independent assortment (below the diagonal) in Vaccinium section Cyanococcus. ${ }^{2}$

\begin{tabular}{|c|c|c|c|c|c|c|c|c|c|c|c|c|c|c|}
\hline & & Acol & $A c 02$ & $A d h 2$ & Ald & G3pdh2 & $I d h$ & Lapl & $M d h 2$ & $M d h 3$ & $6 P g d 1$ & $6 P g d 2$ & Pgi2 & Pgm2 \\
\hline Acol & & & $2(72)$ & $2(68)$ & $\ldots y$ & $1(32)$ & $2(70)$ & $-\cdots$ & 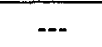 & $\cdots$ & -- & $\ldots$ & $1(64)$ & -.. \\
\hline$A c o 2$ & & NS & & $2(106)$ & $\therefore-$ & $2(118)$ & $2(117)$ & --- & --- & --- & ... & $\ldots$ & $3(167)$ & $\ldots$ \\
\hline Adh2 & & NS & NS & & $2(76)$ & $3(181)$ & $2(143)$ & $-\cdot-$ & $2(82)$ & --- & -.. & $1(62)$ & $4(253)$ & $\cdots$ \\
\hline Ald & & -- & $-\cdots$ & -.. & & $1(96)^{\prime}$ &.- & $1(52)$ & $2(165)$ & --. & -- & $1(88)$ & $3(238)$ & $3(275)$ \\
\hline G3pdh2 & & NS & --. & $1^{*}$ & NS & & $2(187)$ & --- & $\ldots$ & $1(93)$ & $1(91)$ &.-- & $4(355)$ & $1(94)$ \\
\hline$I d h$ & & $\cdots$ & NS & NS & -- & NS & & $\cdots$ & --. & $1(72)$ & -.- & $2(193)$ & $5(430)$ & $1(139)$ \\
\hline Lap1 & & --- & $\cdots$ & $\ldots$ & NS & -- & -- & & $\cdots$ & $2(171)$ & $1(94)$ & $1(68)$ & $3(186)$ & -..- \\
\hline$M d h 2$ & - & $\cdots$ & -- & NS & NS & $-\cdot$ & $\cdots$ & $\cdots$ & & $1(100)$ & -- & $1(63)$ & $2(158)$ & $\cdots$ \\
\hline$M d h 3$ & & --- &.-- & $\cdots$ & $\cdots$ & NS & NS & NS & NS & & $4(394)$ & $2(177)$ & $6(664)$ & $3(292)$ \\
\hline $6 P g d I$ & & -- & -- & $\cdots$ & -- & NS & $\therefore$ & NS & -- & NS & & $1(81)$ & $3(253)$ & $2(152)$ \\
\hline $6 P g d 2$ & & -- & $\ldots$ & $1^{*}$ & NS & $\cdots$ & NS & NS & NS & NS & NS & & $5(424)$ & $2(118)$ \\
\hline Pgi2 & $\because$ & NS & NS & NS & NS & NS & NS & $3^{* * *}$ & NS & NS & $1^{*}$ & NS & & $3(320)$ \\
\hline Pgm2 & & --- & $\ldots$ & --. & $1^{*}$ & NS & NS & $\ldots$ & $\ldots$ & --- & NS & $2^{* * *}$ & NS & \\
\hline
\end{tabular}

${ }^{2}(\mathrm{)}$ Total number of individuals analyzed in all progenies combined.

y---No progenies available jointly segregating for these two loci.

$*, * * *,{ }^{\mathrm{Ns}}$ Number of progenies significant at $P=0.05$ and 0.001 or nonsignificant at $\mathrm{P}=0.05$, respectively.

Table 3. Isozyme loci showing deviations from independent assortment in joint segregation analysis in Vaccinium section Cyanococcus.

\begin{tabular}{lcccc}
\hline \hline Locus pair & Cross & $\begin{array}{c}\text { Progeny with } \\
\text { respective genotype } \\
\text { (no.) }\end{array}$ & $\mathrm{G}$ & $\begin{array}{c}\text { Recombination } \\
\text { value } \pm \text { SE }\end{array}$ \\
\hline Pgi-2/Lap-1 & 6 & $36,1,34,0 / 3,26,3,17$ & $111.77^{* * *}$ & n.d. \\
Lap-1/Pgi-2 & 10 & $31,29,4,0 / 2,4,28,27$ & $120.08^{* * *}$ & n.d. \\
Pgi-2/Lap-1 & 12 & $14,0,13,2 / 1,9,1,12$ & $46.06^{* * *}$ & n.d. \\
6Pgd-2/Pgm-2 & 3 & $11,0 / 6,12$ & $16.42^{* * *}$ & $0.21 \pm 0.07$ \\
6Pgd-2/Pgm-2 & 9 & $25,17,4 / 5,18,20$ & $26.13^{* * *}$ & $0.17 \pm 0.05$ \\
Adh-2/G3pdh-2 & 2 & $25,9 / 15,18$ & $5.57^{*}$ & $0.36 \pm 0.06$ \\
Ald-1/Pgm-2 & 2 & $18,17 / 15,35$ & $3.97^{*}$ & $0.38 \pm 0.05$ \\
Pgi-2/6-Pgd-1 & 6 & $19,15,19,6 / 8,9,9,13$ & $8.31^{*}$ & n.d. \\
6-Pdg-2/Adh-2 & 12 & $26,9 / 13,14$ & $4.48^{*}$ & $0.35 \pm 0.06$ \\
\hline A
\end{tabular}

${ }^{2} \mathrm{~A}$ slash (/) and a comma (,) separate genotypic classes that differ at the first and second locus of the locus pair, respectively; on either side of the slash the sequence of genotypic classes is the same.

*,*** Significant at $P=0.05$ and 0.001 , respectively.

tent with a monomeric structure for this enzyme. A monomeric structure for LAP has been reported in other plant species (Arus et al., 1982; Manosand Fairbrothers, 1987; Suiter, 1988; Wendel and Parks, 1982). The banding positions for Lap-l in the populations analyzed indicated the existence of at least five alleles at Lap-1 (Fig. 1). In addition, the segregation patterns in progenies 6 and 14 revealed the presence of at least one null allele. Segregation ratios for Lap-1 gave mendelian ratios except for cross 6 (Table 1).

Nonsegregating progenies. For all loci discussed, crosses between homozygous genotypes having alleles with identical Rf values exhibited true breeding behaviors: $100 \%$ of the progeny exhibited one band at the respective Rf value, Plants homozygous for different alleles yielded $100 \%$ heterozygotes for those two alleles.

Linkage analyses. Forty-seven pairs of loci were tested for independent assortment (Table 2). Departures from independent assortment (Table 3) revealed linkage for two pairs: Lap-1 with Pgi2 and Pgm-2 with 6-Pgd-2; these were each confirmed in at least two progeny. A few other cases with significant departures $(P \leq$ $0.05)$ from independent assortment occurred: $A d h-2$ with $G-3-p d h$ 2 in cross 2, Ald with Pgm-2 in cross 1, Pgi-2 with 6-Pgd-l in cross 6, and 6-Pgd-2 with $A d h-2$ in cross 12 . In the first three cases, however, these deviations were not confirmed in the other progeny jointly segregating for these pairs of loci.

\section{Discussion}

The mendelian segregation patterns observed at the eight allozyme loci discussed above bring to 13 the number of allozyme loci shown to be inherited as single nuclear genes in blueberry. The observed number of aberrant segregations constitute $6 \%$ of the 65 segregation ratios tested, if segregations for $I d h, M d h$ 3, 6-Pgd-2, and Pgi-2, not shown in Table 1, are included. This is about the number of distorted segregations one would expect to observe due to chance as a Type I error considering the 0.05 level of significance chosen. Two crosses, 2 and 9, could account for most of the deviations from mendelian segregation (Table 1). Cross 9 is a cross between half-siblings and it is possible that the parents in cross 2 also share common ancestry. This situation may have contributed to segregation distortion through the unmasking of any deleterious recessive genes linked to allozyme loci.

The two linkage groups found, Lap-1/Pgi-2 and 6-Pgd-2/Pgm2 , appeared to be independent from one another as no significant 
deviations from independent assortment between 6-Pgd-2 and Pgi-2, Pgi-2 and Pgm-2, or 6-Pgd-2 and Lap-1 were found. These two pairs of linked loci are the first to be reported in blueberry. Linkage between loci coding for the same pairs of enzyme systems has been found in a variety of plant species: Gpi-c and Lap-1 in grape (Weeden et al., 1988) and Pgi-1 and Lap-2 in cotton (Suiter, 1988), Pgd-p and Pgm-c in pea (Weeden and Marx, 1987), 6-Pgd-1 and Pgm-2 in tomato (Tanksley, 1985), 6-Pgd-2 and Pgm-2 in Camellia japonica (Wendel and Parks, 1982), and Pgd-p and Pgm-c in lentil (Muehlbauer et al., 1989). Although these taxa are highly divergent, these could possibly represent regions of linkage conservation. First, however, the genes involved would need to be proven homologous. One method for analyzing relationships is to compare subcellular locations of isozymes. An initial effort to determine subcellular localization of the enzymes by using pollen leachates (Weeden and Gottlieb, 1980) was unsuccessful; pollen leachates did not exhibit activity for any of the enzymes. Also, the linkages reported here were determined in the $V$. darrowi and $V$. elliottii species only and should be investigated in other Vaccinium species as well.

The three deviations from independent assortment for $A d h-2 /$ G-3-pdh-2, Ald/Pgm-2, and Pgi-2/6-Pgd-1 do not allow us to confirm linkage yet as they were not significant in two of the three progenies jointly segregating for these loci. The analysis of more progenies involving different parents should indicate whether these deviations represent simple random variation or weakly linked loci. As there was only one progeny jointly segregating for $6-P g d-2 / A d h-2$, the analysis of more progenies would be desirable to ascertain whether its deviation from independent assortment for these pairs of loci can be confirmed.

\section{Literature Cited}

Arus, P., S.D. Tanksley, T.J. Orton, and R.A. Jones. 1982. Electrophoretic variation as a tool for determining seed purity and for breeding hybrid varieties of Brassica oleracea. Euphytica 31:417-428.

Bruederle, L.P. and N. Versa. 1989. Population genetic structure in diploid blueberry, Vaccinium § Cyanococcus A. Gray (Ericaceae). Amer. J. Bet. 76:229. (Abstr.)

Bruederle, L.P. and N. Versa. 1990. Systematic relationships among the diploid blueberry species (Vaccinium § Cyanococcus A. Gray; Ericaceae). Amer. J. Bet. 77:122.

Bruederle, L. P., N. Versa, and J.R. Ballington. 1991. Population genetic structure in diploid blueberry, Vaccinium $\S$ cyanococcus (Ericaceae). Amer. J. Bet. (In press.)

Clayton, JW. and D.N. Tretiak. 1972. Amine-citrate buffers for $\mathrm{pH}$ control in starch gel electrophoresis. J. Fish. Res. Board Can. 29: 11691172.

Griffin, J.D. and R.G. Palmer. 1987. Inheritance and linkage studies with five isozyme loci in soybean. Crop Sci. 27:885-892.

Hall, S.H. and G.J. Galletta. 1971. Comparative chromosome morphology of diploid Vaccinium species. J. Amer. Soc. Hort. Sci. 96:289-292.

Hyun, J. O., O.J. Rajora, and L. Zsuffa. 1987. Inheritance and linkage of isozymes in Populus tremuloides (Michx.). Genome 29:384-388.

Krebs, S.L. and J.F. Hancock. 1989. Tetrasomic inheritance of isozyme markers in the highbush blueberry, Vaccinium corymbosum L. Heredity 63:11-18, 1988.

Longo, G.P. and J.G. Scandalios. 1969. Nuclear gene control of mitochondrial malic dehydrogenase in maize. Proc. Natl. Acad. Sci. 62:104-111.

Manes, P.S. and D.E. Fairbrothers. 1987. Allozyme variation in populations of six Northeastern American red oaks. Systematic Bet. 12:365-373.

Muehlbauer, F. J., N.F. Weeden, and D.L. Hoffman. 1989. Inheritance and linkage relationships of morphological and isozyme loci in lentil (Lens Miller). J. Hered. 80:298-303.

Sokal, R.R. and F.J. Rohlf. 1981. Biometry. W.H. Freeman, New York.

Soltis, D. E., C.H. Haufler, D.C. Darrow, and G.J. Gastony. 1983. Starch gel electrophoresis of ferns: a compilation of grinding buffers, gels and electrode buffers, and staining schedules. Amer. Fern J. 73:9-27.

Sternberg, L. and I.P. Ting. 1979. Inheritance of microbody malate dehydrogenase in Opuntia basilaris. J. Hered. 70:220.

Suiter, K.A. 1988. Genetics of allozyme variation in Gossypium arboreum L. and Gossypium herbaceum L. (Malvaceae). Theor. Applied Genet. 75:259-271.

Suiter, K. A., J.F. Wendel, and J.S. Case. 1983. LINKAGE-1: a PASCAL computer program for the detection and analysis of genetic linkage. J. Hered. 74:203-204.

Tanksley, S.D. 1985. Enzyme coding genes in tomato (Lycopersicon esculentum). Isozyme Bul. 18:43-45.

Vander Kloet, S.P. 1988. The genus Vaccinium in North America. Research Branch. Agr. Can. Ottawa.

Versa, N., P.S. Manes, and M.I. van Heemstra. 1988. Isozyme variation and inheritance in blueberry. Genome 30:776-781.

Weeden, N.F. and L.D. Gottlieb. 1980. Isolation of cytoplasmic enzymes from pollen. Plant Physiol. 66:400-403.

Weeden, N.F. and G.A. Marx. 1987. Further genetic analysis and linkage relationships of isozyme loci in the pea. J. Hered. 78:153159.

Weeden, N. F., B.I. Reisch, and M.E. Martens. 1988. Genetic analysis of isozyrne polymorphism in grape. J. Amer. Soc. Hort. 113:765769.

Wendel, J.F. and C.R. Parks. 1982. Genetic control of isozyme variation in Camellia japonica L. J. Hered. 73:197-204. 